\title{
Prevalência de obesidade em crianças de uma escola pública e de um ambulatório geral de Pediatria de hospital universitário
}

\author{
Obesity prevalence among students of a public school and a Pediatric out-patient clinic of a university hospital
}

Júlia Kefalás Troncon ${ }^{1}$, Juliana Pierobon Gomes ${ }^{1}$, Gil Guerra-Júnior², Cristina Alba Lalli

\section{RESUMO}

Objetivo: Verificar a associação entre atividade física e características socioeconômicas com a presença de obesidade e/ou sobrepeso em crianças de seis a 14 anos, escolares da Escola Sérgio Porto e pacientes que procuraram o Ambulatório de Pediatria no Hospital de Clínicas (HC), ambos no campus da Universidade Estadual de Campinas (Unicamp).

Métodos: $\mathrm{O}$ estudo foi realizado por meio da aplicação de questionário aos pais ou responsáveis e coleta de dados de peso e altura das crianças. Foi calculado o índice de massa corpórea (IMC), e feita a classificação em normal, sobrepeso ou obesidade, a partir dos dados do Centers for Disease Control and Prevention (CDC), dos Estados Unidos. Foi feita análise descritiva dos dados e utilizados os testes do qui-quadrado ou exato de Fisher.

Resultados: A amostra ambulatorial foi composta por 107 crianças $(13,1 \%$ com sobrepeso e $11,2 \%$ obesas) e a escolar de 109 ( $16,5 \%$ com sobrepeso e 20,2\% obesas). Não foi observada diferença significante entre a prevalência de obesidade ou de obesidade e sobrepeso entre as duas amostras, apesar de as amostras serem diferentes em relação à renda mensal $(p<0,0001)$, escolaridade materna $(p<0,0001)$ e atividade física $(p<0,0001)$, sendo que a população ambulatorial apresentou menores índices nestas três variáveis, comparada à escolar.

Conclusões: Os índices de sobrepeso e obesidade encontrados no presente estudo foram elevados, confirmando que houve a transição nutricional em ambas as amostras, de maneira similar à observada no resto do nosso país, independentemente do sexo, da classificação socioeconômica e da atividade física.

Palavras-chave: obesidade; criança; prevalência; atividade física.

\section{ABSTRACT}

Objective: The aim of this study was to verify the association between physical activity and socio-economic status with the presence of obesity and/or overweight in children aged six to 14 years of two different sources: an elementary school and an out-patient Pediatric clinic of one university hospital of Campinas, São Paulo.

Methods: A socio-demographic questionnaire was answered by parents and the evaluation of children's weight and height was performed according to Centers for Disease Control and Prevention (CDC), USA, standards for body mass index (BMI) The children were divided in three categories: normal, overweight and obese. Chisquare and Fisher exact test were used to compare both groups of children.

Results: 107 children from the university hospital were studied $(13.1 \%$ with overweight and $11.2 \%$ obese) as well as 109 from the elementary school (16.5\% overweight and $20.2 \%$ obese). Both groups were similar regarding the prevalence of obesity alone or obesity and overweight. Groups differed in family income $(p<0.0001)$, number of maternal years in school $(p<0.0001)$ and physical activity $(p<0.0001)$, with disadvantage of the clinic patients.

Conclusions: The frequency of overweight and obesity observed in this study were high, pointing out that a nutritional transition has occurred in both samples independently of gender, socio-economic status and physical activity.

Key-words: obesity; child; prevalence; motor activity.
${ }^{1}$ Aluno de Medicina da Universidade Estadual de Campinas (Unicamp), Campinas, SP, Brasil

2Professor associado do Departamento de Pediatria da Faculdade de Ciências Médicas (FCM) da Unicamp, Campinas, SP, Brasil

${ }^{3}$ Médica do Hospital de Clínicas emestre em Clínica Médica daFCM-Unicamp

Campinas, SP, Brasil
Endereço para correspondência:

Cristina Alba Lalli

Rua Barão de Paranapanema, 222/73 - Bosque

CEP 13026-010 - Campinas/SP

E-mail: clalli@c.unicamp.br

Recebido em: 22/3/2007

Aprovado em: 20/8/2007 


\section{Introdução}

A obesidade é definida como o excesso de gordura corporal em relação à massa magra, sendo uma doença cuja prevalência vem aumentando nas últimas décadas, tanto em adultos como em crianças; hoje, já é considerada uma pandemia. Dados do International Obesity Task Force (IOTF) indicam que, no mundo, 22 milhões de crianças com menos de cinco anos têm sobrepeso ${ }^{(1)}$.

A prevalência elevada de obesidade é um grave problema de saúde pública, uma vez que outras doenças e síndromes estão a ela associadas, com conseqüente redução da expectativa de vida ${ }^{(2)}$. Ainda, dentre as inúmeras conseqüências da obesidade, sabe-se que $60 \%$ das crianças de seis a 12 anos que estão acima do peso têm ao menos um fator de risco para doenças cardiovasculares ${ }^{(3)}$ e que o dano à parede arterial ocasionado na infância devido ao excesso de peso é permanente ${ }^{(4)}$.

Em relação à patogênese, vários estudos têm sido realizados, abordando aspectos comportamentais e ambientais, além de avaliar aspectos genéticos e metabólicos. Pesquisas relacionadas a aspectos comportamentais sugerem que a obesidade é resultado do excesso de ingestão calórica e do sedentarismo; entretanto, em seus resultados preliminares, o National Health and Nutrition Examination Survey (NHANES) demonstrou que a ingestão calórica havia mudado pouco nas crianças e adolescentes avaliados ${ }^{(5)}$. $\mathrm{O}$ gasto energético por meio da atividade física é um elemento importante na avaliação causal da obesidade; porém, estudos recentes sugerem que a inatividade possa ser mais uma conseqüência do que uma de suas causas ${ }^{(5,6)}$.

Em relação ainda ao componente ambiental, estudos comprovaram que os pais têm importante papel na determinação das preferências alimentares da criança - principalmente nos primeiros dois anos de vida - e são também fundamentais na construção da auto-estima e da auto-imagem ${ }^{(7)}$.

No que se refere ao componente genético, estudos realizados com crianças adotadas mostraram que estas possuíam índice de massa corpórea (IMC) relacionada com o de seus pais biológicos e, não, com o dos adotivos. Essa descoberta indica que, embora o ambiente exerça papel importante na determinação da obesidade, a influência genética também é crucial para o seu desenvolvimento(4).

O US Pediatric Nutrition Surveillance System (PedNSS) evidenciou que crianças com baixo peso ao nascer possuíam maior risco relativo de permanecerem obesas em idade pré-escolar, após terem se tornado obesas nos dois primeiros anos de vida, quando comparadas a crianças que já apresentavam peso normal ou elevado ao nascer ${ }^{(8)}$. Uma hipótese para explicar tal fato seria o catch-up growth, que consiste no crescimento rápido pós-natal, recuperando o peso e a altura da criança, o que resultaria em obesidade infantil - a qual permanece na adolescência e na idade adulta e tem sido relacionada ao maior risco de desenvolvimento de diabetes tipo $2^{(9-11)}$.

Segundo o Consenso Latino-Americano em Obesidade, a situação emergente da obesidade nos países em desenvolvimento é particularmente crítica e afeta não somente os grupos economicamente favorecidos, mas também os menos favorecidos. Ainda que a prevalência seja substancialmente menor do que nos países desenvolvidos, o número total de pessoas afetadas é proporcionalmente maior. A informação disponível para a América Latina na década de 1980 a 1990 reflete prevalência de sobrepeso no Chile de $50 \%$ para as mulheres e $40 \%$ para os homens; no Brasil, de $40 \%$ para as mulheres e $27 \%$ para os homens; e, no México, de 30\% para os homens e $35 \%$ para as mulheres ${ }^{(12)}$.

No Brasil, Sotelo et a ${ }^{(13)}$, avaliando 2.519 escolares no projeto Redução dos Riscos de Adoecer e Morrer na Maturidade (RRAMM) e utilizando três critérios diferentes para diagnóstico de sobrepeso e obesidade, encontraram valores entre 10,29 e $11,94 \%$ de sobrepeso e 8,17 e $13,67 \%$ de obesidade.

O objetivo deste estudo foi verificar a associação entre atividade física e características socioeconômicas com a presença de obesidade e/ou sobrepeso em crianças de seis a 14 anos em duas amostras populacionais diferentes: escolares que freqüentavam a Escola Estadual Sérgio Porto, que atende a filhos de funcionários da Universidade Estadual de Campinas (Unicamp), e pacientes que procuraram o Ambulatório de Pediatria no Hospital de Clínicas (HC), ambos situados no campus da Unicamp, na cidade de Campinas, interior de São Paulo.

\section{Métodos}

Trata-se de um estudo do tipo seccional, com aplicação de um questionário semi-estruturado elaborado pelo grupo de pesquisa. A partir desse questionário, foram obtidos dados como sexo, escolaridade e profissão dos pais ou responsáveis. O projeto foi aprovado pelo Comitê de Ética em Pesquisa da Faculdade de Ciências Médicas (FCM) da Unicamp.

Como a pesquisa foi realizada em duas vertentes, a aplicação do questionário se fez de forma diferente nas duas amostras. Na Escola Estadual Sérgio Porto, dentre um total de 355 crianças que cursavam da primeira à quarta série e com idade entre seis e 14 anos, o questionário e o consentimento 
informado foram entregues aos alunos do período vespertino, totalizando 250 crianças. $\mathrm{O}$ pesquisador compareceu às salas de aula, proferindo breve explicação sobre a pesquisa e sobre o preenchimento dos dados. Os questionários foram então preenchidos pelos pais ou responsáveis dos alunos em seus domicílios, retornando aos professores três dias após a entrega. Dos questionários entregues, 141 foram excluídos: 100 por não terem retornado em mãos do pesquisador e 41 porque não apresentavam preenchimento correto de dados ou por não apresentarem o consentimento livre esclarecido assinado de forma correta. O número de questionários resultantes para essa amostra foi 109 (amostra escola). Todas as crianças selecionadas dentro dos critérios supracitados foram pesadas e medidas pelo pesquisador. Essas também foram entrevistadas para esclarecer possíveis dúvidas a respeito do preenchimento do questionário.

No Ambulatório de Pediatria do HC-Unicamp, a amostra foi composta por pacientes de seis a 14 anos que haviam procurado o serviço por outros motivos que não a obesidade, escolhidos aleatoriamente na sala de espera. A seleção foi feita pelo pesquisador por meio de verificação da idade no prontuário do paciente. Os dados de peso e altura foram obtidos pela equipe de enfermagem. Os pacientes e seus responsáveis foram submetidos à aplicação do questionário pelo próprio pesquisador, após assinatura de consentimento informado. O total desta amostra foi de 107 pacientes (amostra ambulatório).

A partir dos dados de peso e altura, foi calculado o IMC $\left(\mathrm{IMC}=\right.$ peso em $\mathrm{kg} /$ altura em $\mathrm{m}^{2}$ ) e utilizados os gráficos elaborados pelo Centers of Disease Control and Prevention (CDC) norte-americano, segundo idade e sexo, para classificar as crianças em normais (IMC abaixo do percentil 85), com sobrepeso (IMC entre os percentis 85 e 95) ou obesas (IMC acima do percentil 95).

Para a classificação social e econômica, foi utilizada a renda mensal familiar em salários-mínimos (dividida em: sem renda, de zero a dois salários-mínimos (SM), de dois a cinco, de cinco a dez, de dez a 20, e sem informação), a escolaridade materna (dividida em analfabeta, primeiro grau, segundo grau, superior incompleto ou completo, e sem informação) e o esquema de divisão em classes sociais e frações de classe com base no proposto por Singer ${ }^{(14)}$ e adaptado por Barros ${ }^{(15)}$ a partir da informação da profissão e ocupação do responsável de maior escolaridade (dividida em subproletariado, proletariado propriamente dito, burguesia gerencial, pequena burguesia, burguesia empresarial e sem informação). A atividade física foi classificada em: sedentária (sem nenhuma atividade, inclusive brincar fora de casa); atividade leve (com atividade de até duas vezes na semana, em geral atividade curricular); moderada (atividade curricular mais atividade esportiva extracurricular duas a três vezes na semana); e intensa (atividade curricular mais atividade esportiva de quatro vezes por semana ou mais).

Os dados foram armazenados em planilha do SPSS versão 10.0. Foi feita análise descritiva dos dados com criação de tabelas e utilizado o programa Epi-Info 6 para o cálculo do qui-quadrado ou teste exato de Fisher, com nível de significância de $5 \%$.

\section{Resultados}

Das 107 crianças avaliadas na amostra ambulatorial, 14 apresentaram sobrepeso $(13,1 \%)$ e 12 , obesidade $(11,2 \%)$. Em relação à classificação por diagnóstico, dos 109 escolares, observou-se que 18 crianças apresentavam sobrepeso $(16,5 \%)$ e 22 eram obesas $(20,2 \%)$. Não foi observada diferença estatística entre as amostras ambulatório e escola em relação à prevalência de obesidade isolada $(p=0,09)$ e obesidade associada ao sobrepeso $(p=0,07)$, conforme Tabela 1 .

Em relação ao sexo, não houve diferença entre as amostras $(p=0,50)$. A amostra ambulatório apresentou $54,2 \%$ de sexo feminino e a amostra escola, 59,6\%. Também não foi observada associação significativa entre sexo e estado nutricional nas duas amostras, quer avaliando-se a obesidade isoladamente (amostra ambulatório: $p=0,57$; amostra escola: $p=0,44$ ), quer avaliando-se a obesidade e o sobrepeso associados (amostra ambulatório: $p=0,85$; amostra escola: $p=0,58)$, como mostra a Tabela 1 .

Tabela 1 - Distribuição do estado nutricional de acordo com o sexo

\begin{tabular}{lcccccc}
\hline & \multicolumn{2}{c}{ Obesidade } & \multicolumn{2}{c}{ Sobrepeso } & \multicolumn{2}{c}{ Normal } \\
& Ambulatório & Escola & Ambulatório & Escola & Ambulatório & Escola \\
\hline Feminino & $8(66,6 \%)$ & $11(50,0 \%)$ & $7(50,0 \%)$ & $11(61,1 \%)$ & $43(53,1 \%)$ & $43(62,3 \%)$ \\
Masculino & $4(33,3 \%)$ & $11(50,0 \%)$ & $7(50,0 \%)$ & $7(38,8 \%)$ & $38(46,9 \%)$ & $26(37,7 \%)$ \\
Total & $12(100 \%)$ & $22(100 \%)$ & $14(100 \%)$ & $18(100 \%)$ & $81(100 \%)$ & $69(100 \%)$ \\
\hline
\end{tabular}


Em relação à renda familiar declarada, foi observada diferença estatisticamente significante entre as amostras quando comparados os grupos de renda até dois SM, versus o grupo de dois a cinco SM e versus aqueles com renda acima de cinco SM, sendo menor a renda na amostra ambulatório $(p<0,0001)$. No entanto, quando verificada a associação entre estado nutricional e renda familiar com os mesmos agrupamentos em cada amostra, não foi observada associação significante, quer avaliando-se a obesidade isoladamente (amostra ambulatório: $p=0,78$; amostra escola: $p=0,41$, quer avaliando-se a obesidade e o sobrepeso associados (amostra ambulatório: $p=0,92$; amostra escola: $p=0,46)$, de acordo com a Tabela 2 .

Em relação à escolaridade da mãe, foi observada diferença estatisticamente significante entre as amostras, quando comparados os grupos analfabeta e primeiro grau incompleto versus primeiro grau completo e segundo grau incompleto versus o segundo grau completo e superior incompleto ou completo, sendo menor a escolaridade materna na amostra ambulatório $(p<0,0001)$. No entanto, quando verificada a associação entre o estado nutricional e a escolaridade materna com os mesmos agrupamentos em cada amostra, não foi observada associação significativa, quer avaliando-se a obesidade isoladamente (amostra ambulatório: $p=0,14 ; p=0,93$; amostra escola: $p=0,35)$, quer avaliando-se a obesidade e o sobrepeso associados ( $p=0,61$; amostra escola: $p=0,09)$, conforme Tabela 3 .

Quanto à ocupação do responsável de maior escolaridade, não foi observada diferença estaticamente significativa entre as amostras quando comparados os grupos subproletariado e proletariado propriamente dito versus burguesia gerencial versus pequena burguesia e burguesia empresarial $(p=0,54)$. No entanto, quando verificada a associação entre o estado nutricional e a ocupação do responsável de maior escolaridade com os mesmos agrupamentos em cada amostra, foi observada associação significativa apenas na amostra ambulatório para a obesidade isoladamente, com menor prevalência de obesidade nos grupos subproletariado e proletariado propriamente dito $(p=0,02)$. Não foi observada tal associação para a amostra escola (Fisher; $p=1,00)$, assim como não houve associação entre ocupação e sobrepeso em nenhum dos dois grupos (amostra ambulatório: $p=0,13$; amostra escola: Fisher $=0,29)$, de acordo com a Tabela 4.

Quanto à atividade física, foi observada diferença estaticamente significante entre as amostras quando comparados os grupos sedentário e atividade leve versus atividade moderada e intensa, com atividade física mais importante na amostra escola

Tabela 2 - Distribuição do estado nutricional de acordo com a renda mensal familiar em salários-mínimos (SM)

\begin{tabular}{lcccccc}
\hline & \multicolumn{2}{c}{ Obesidade } & \multicolumn{2}{c}{ Sobrepeso } & \multicolumn{2}{c}{ Normal } \\
& Ambulatório & Escola & Ambulatório & Escola & Ambulatório & Escola \\
\hline Sem renda & 0 & 0 & $1(7,1 \%)$ & 0 & $1(1,2 \%)$ & 0 \\
0 a 2 SM & $4(33,3 \%)$ & $2(9,1 \%)$ & $5(35,7 \%)$ & $1(5,6 \%)$ & $27(33,3 \%)$ & $6(8,7 \%)$ \\
2 a 5 SM & $5(41,7 \%)$ & $5(22,7 \%)$ & $7(50,0 \%)$ & $7(38,9 \%)$ & $41(50,6 \%)$ & $27(39,1 \%)$ \\
5 a 10 SM & $2(16,7 \%)$ & $9(40,9 \%)$ & $1(7,1 \%)$ & $7(38,9 \%)$ & $8(9,9 \%)$ & $20(29,0 \%)$ \\
10 a 20 SM & 0 & $1(4,5 \%)$ & 0 & $1(5,6 \%)$ & $1(1,2 \%)$ & $3(4,3 \%)$ \\
Sem informação & $1(8,3 \%)$ & $5(22,7 \%)$ & 0 & $2(11,1 \%)$ & $3(3,7 \%)$ & $13(18,8 \%)$ \\
Total & $12(100 \%)$ & $22(100 \%)$ & $14(100 \%)$ & $18(100 \%)$ & $81(100 \%)$ & $69(100 \%)$ \\
\hline
\end{tabular}

Tabela 3 - Distribuição do estado nutricional de acordo com a escolaridade da mãe

\begin{tabular}{lcccccc}
\hline & \multicolumn{2}{c}{ Obesidade } & \multicolumn{2}{c}{ Sobrepeso } & \multicolumn{2}{c}{ Normal } \\
& Ambulatório & Escola & Ambulatório & Escola & Ambulatório & Escola \\
\hline Analfabeto & 0 & 0 & 0 & 0 & $1(1,2 \%)$ & $1(1,4 \%)$ \\
$1^{\circ}$ grau incompleto & $6(54,5 \%)$ & $1(4,5 \%)$ & $6(42,9 \%)$ & $1(5,6 \%)$ & $39(47,6 \%)$ & $3(4,3 \%)$ \\
$1^{\circ}$ grau completo & $1(9,1 \%)$ & $1(4,5 \%)$ & $2(14,3 \%)$ & $4(22,2 \%)$ & $15(18,3 \%)$ & $2(2,9 \%)$ \\
$2^{\circ}$ grau incompleto & $1(9,1 \%)$ & $5(22,7 \%)$ & $3(21,4 \%)$ & $1(5,6 \%)$ & $1(1,2 \%)$ & $6(8,7 \%)$ \\
$2^{\circ}$ grau completo & $3(27,3 \%)$ & $8(36,4 \%)$ & $2(14,3 \%)$ & $8(44,4 \%)$ & $17(20,7 \%)$ & $39(56,5 \%)$ \\
Superior incompleto & 0 & $3(13,6 \%)$ & 0 & $1(5,6 \%)$ & $4(4,9 \%)$ & $7(10,1 \%)$ \\
Superior completo & 0 & $2(9,1 \%)$ & 0 & $2(11,1 \%)$ & $1(1,2 \%)$ & $7(10,1 \%)$ \\
Sem informação & 0 & $2(9,1 \%)$ & $1(7,1 \%)$ & $1(5,6 \%)$ & $4(4,8 \%)$ & $4(5,8 \%)$ \\
Total & $11(100 \%)$ & $22(9,1 \%)$ & $14(100 \%)$ & $18(100 \%)$ & $82(100 \%)$ & $69(100 \%)$ \\
\hline
\end{tabular}


$(p<0,0001)$. No entanto, quando verificada a relação entre o estado nutricional e a atividade física, foi observada associação significativa apenas na amostra escola, com maior prevalência de obesidade e sobrepeso nos grupos com maior atividade física (Fisher=0,006). Não foi observada associação entre atividade física e obesidade isoladamente para a amostra ambulatório $(p=0,18)$ e para a amostra escola (Fisher; $p=0,11)$ e também não foi notada associação entre obesidade e sobrepeso com a atividade física para a amostra ambulatório $(p=0,37)$, segundo a Tabela 5 .

\section{Discussão}

Ainda que alguns estudos sugiram que o sexo feminino possa ser fator de risco para a obesidade infantil ${ }^{(16)}$, esse dado não é um consenso e nossos resultados não mostraram diferença significante na prevalência de alterações do estado nutricional em relação ao sexo.

Quanto à atividade física, há controvérsias na literatura se o sedentarismo é causa de excesso de peso em função do menor gasto energético ou é conseqüência, uma vez que a obesidade leva a pessoa a ter menos disposição e auto-estima, tornando-se mais inativa fisicamente ${ }^{(5,6)}$. O Framingham Children's Study acompanhou 106 crianças da infância à adolescência e concluiu que a taxa de obesidade em crianças que assistem a mais de cinco horas de televisão por dia pode chegar a ser até 8,3 vezes maior do que para aquelas que assistem duas ou menos horas diárias de televisão ${ }^{(17)}$. Em nosso estudo, foi observada prática de atividade física mais freqüente na amostra escola. Surpreendentemente, no entanto, nesse grupo foi encontrada a maior freqüência de atividade física nos indivíduos classificados como sobrepeso e obesos. Esse achado pode refletir a melhor condição socioeconômica e cultural do grupo, com maior estímulo às práticas esportivas extra-escolares e acesso à orientação profissional relativa ao excesso de peso.

As duas amostras avaliadas no presente estudo apresentaram diferenças significativas em relação aos parâmetros sociais. Os alunos que freqüentam a Escola Estadual Sérgio Porto, em sua maioria, pertencem a famílias em que pelo menos um dos pais é funcionário da Universidade, que exige no mínimo escolaridade média de seus colaboradores. Portanto, essa amostra apresentou média maior de escolaridade materna e maior média salarial, comparada às famílias que procuraram o ambulatório do hospital público. A relação entre a renda familiar e a prevalência de alterações do peso em crianças também apresenta aspectos contraditórios. Dados da Pesquisa de Orçamentos Familiares (POF) 2002-2003 do Instituto Brasileiro de Geografia e Estatística (IBGE) ${ }^{(18)}$ demonstraram que a porcentagem de indivíduos com sobrepeso e obesos aumenta à medida que aumenta a renda familiar. Em estudo que avaliou o

Tabela 4 - Distribuição do estado nutricional de acordo com a ocupação do responsável de maior escolaridade

\begin{tabular}{|c|c|c|c|c|c|c|}
\hline & \multicolumn{2}{|c|}{ Obesidade } & \multicolumn{2}{|c|}{ Sobrepeso } & \multicolumn{2}{|l|}{ Normal } \\
\hline & Ambulatório & Escola & Ambulatório & Escola & Ambulatório & Escola \\
\hline Subproletariado & $4(33,3 \%)$ & $1(4,5 \%)$ & $10(71,4 \%)$ & 0 & $52(64,2 \%)$ & $2(2,9 \%)$ \\
\hline Proletariado & $7(58,3 \%)$ & $13(59,1 \%)$ & $3(21,4 \%)$ & $14(77,8 \%)$ & $23(28,4 \%)$ & $50(72,5 \%)$ \\
\hline Burguesia gerencial & 0 & 0 & 0 & 0 & $3(3,7 \%)$ & $4(5,8 \%)$ \\
\hline Pequena burguesia & $1(8,3 \%)$ & 0 & 0 & 0 & 0 & 0 \\
\hline Burguesia empresarial & 0 & 0 & 0 & 0 & 0 & 0 \\
\hline Sem informação & 0 & $8(36,4 \%)$ & $1(7,1 \%)$ & $4(22,2 \%)$ & $3(3,7 \%)$ & $13(18,8 \%)$ \\
\hline Total & $12(100 \%)$ & $22(100 \%)$ & $14(100 \%)$ & $18(100 \%)$ & $81(100 \%)$ & $69(100 \%)$ \\
\hline
\end{tabular}

Tabela 5 - Distribuição do estado nutricional de acordo com a atividade física do escolar

\begin{tabular}{lcccccc}
\hline & \multicolumn{2}{c}{ Obesidade } & \multicolumn{2}{c}{ Sobrepeso } & \multicolumn{2}{c}{ Normal } \\
& Ambulatório & Escola & Ambulatório & Escola & Ambulatório & Escola \\
\hline Sedentário & $2(16,7 \%)$ & 0 & 0 & 0 & $11(13,6 \%)$ & $10(14,5 \%)$ \\
Leve & $5(41,7 \%)$ & 0 & $11(78,6 \%)$ & 0 & $54(66,7 \%)$ & $1(1,4 \%)$ \\
Moderada & $5(41,7 \%)$ & $20(91,0 \%)$ & $1(7,1 \%)$ & $16(88,9 \%)$ & $9(11,1 \%)$ & $41(59,4 \%)$ \\
Intensa & 0 & $2(9,1 \%)$ & $2(14,3 \%)$ & $2(11,1 \%)$ & $7(8,6 \%)$ & $17(24,6 \%)$ \\
Total & $12(100 \%)$ & $22(100 \%)$ & $14(100 \%)$ & $18(100 \%)$ & $81(100 \%)$ & $69(100 \%)$ \\
\hline
\end{tabular}


estado nutricional de 387 alunos de escolas públicas e particulares de Salvador, Bahia, com idades entre cinco e 10 anos observou-se maior prevalência de obesidade (30\%) nas escolas particulares em relação às escolas públicas $(8 \%)(p<0,001)^{(19)}$. Confirmando esse dado, Escrivão et $a l^{(20)}$ avaliaram os resultados dos inquéritos nutricionais nacionais e demonstraram que a prevalência de obesidade na região Sul $(7,2 \%)$ e Sudeste $(6,2 \%)$ era mais que o dobro da observada na região Nordeste (2,5\%), ficando as regiões Norte e Centro-Oeste em situação intermediária. Nova avaliação dos dados dos mesmos inquéritos concluiu que a prevalência de obesidade aumenta de acordo com o aumento da renda familiar per capita ${ }^{(21)}$. Já em um estudo que analisou o estado socioeconômico e o risco de obesidade em crianças de escolas públicas do estado de Virgínia, nos
Estados Unidos, a menor renda familiar foi fator de risco para obesidade ${ }^{(22)}$. Esses dados demonstram a diferença entre os países desenvolvidos e em desenvolvimento, sendo a obesidade relacionada à menor renda nos primeiros e à maior renda nos últimos. No entanto, não encontramos associação estatisticamente significante entre a prevalência de obesidade e sobrepeso nas duas amostras em relação à escolaridade materna e à renda familiar.

Apesar de ambas as populações estudadas serem pouco representativas da população brasileira geral, podemos concluir que os índices de sobrepeso e obesidade encontrados estão elevados, de forma semelhante a outros estudos, e, apesar das diferenças sociais e econômicas entre as duas amostras, não foi observada diferença entre as prevalências de obesidade e sobrepeso entre elas.

\section{Referências bibliográficas}

1. Deitel M. The International Obesity Task Force and "globesity". Obes Surg 2002;12:613-4.

2. Friedman JM. Obesity in the new millennium. Nature 2000;404:632-4.

3. Steinberger J, Daniels SR. Obesity, insulin resistance, diabetes and cardiovascular risk in children: an American Heart Association scientific statement from the Atherosclerosis, Hypertension, and Obesity in the Young Committee (Council on Cardiovascular Disease in The Young) and the Diabetes Committee (Council on Nutrition, Physical Activity, and Metabolism). Circulation 2003;107:1448-53.

4. Miller J, Rosenbloom A, Silverstein J. Childhood obesity. J Clin Endocrinol Metab 2004;89:4211-8.

5. Slyper AH. The pediatric obesity epidemic: causes and controversies. J Clin Endocrinol Metab 2004;89:2540-7.

6. Salbe AD, Weyer C, Harper I, Lindsay RS, Ravussin E, Tataranni PA. Assessing risk factors for obesity between childhood and adolescence: II. Energy metabolism and physical activity. Pediatrics 2002;110:307-14.

7. Golan M, Crow S. Parents are key players in the prevention and treatment of weight-related problems. Nutr Rev 2004;62:39-50.

8. Mei Z, Grummer-Strawn LM, Scanlon KS. Does overweight in infancy persist through the preschool years? An analysis of CDC Pediatric Nutrition Surveillance System data. Soz Praventivmed 2003;48:161-7.

9. Hack M, Schluchter M, Cartar L, Rahman M, Cuttler L, Borawski E. Growth of very low birth weight infants to age 20 years. Pediatrics 2003; 112:e30-8.

10. Lundgren EM, Cnattingius S, Jonsson B, Tuvemo T. Prediction of adult height and risk of overweight in females born small-for-gestational-age. Paediatr Perinat Epidemiol 2003;17:156-63.

11. Veening MA, Van Weissenbruch MM, Delemarre-Van De Waal HA. Glucose tolerance, insulin sensitivity and insulin secretion in children born small for gestational age. J Clin Endocrinol Metab 2002;87:4657-61.
12. Coutinho W, editor. Documento do consenso latino-americano sobre obesidade [monograph on the internet] Rio de Janeiro: ABESO; 1998 [cited 2007 Aug 10]. Available from: http://www.abeso.org.br/pdf/consenso.pdf

13. Sotelo YOM, Colugnati FAB, Taddei JAAC. Prevalence of overweight and obesity in public school pupils according to three anthropometric diagnostic criteria. Cad Saúde Publ 2004;20:233-40.

14. Singer PI. Dominação e desigualdade: estrutura de classes e repartição da renda no Brasil - estudos brasileiros, 49. Rio de Janeiro: Paz e Terra; 1981

15. Barros MB. A utilização do conceito de classe social nos estudos dos perfis epidemiológicos: uma proposta. Rev Saude Publ 1986;20:269-73.

16. Martin KS, Ferris AM. Food insecurity and gender are risk factors for obesity. J Nutr Educ Behav 2007;39:31-6.

17. Proctor MH, Moore LL, Gao D, Cupples LA, Bradlee ML, Hood MY, et al. Television viewing and change in body fat from preschool to early adolescence: The Framingham Children's Study. Int J Obes Relat Metab Disord 2003;27:827-33.

18. IBGE [homepage on the Internet]. Pesquisa de orçamentos familiares: antropometria e análise do estado nutricional de crianças e adolescentes no Brasil 2002-2003 [cited 2007 Aug 10]. Available from: http://www.ibge. gov.br/home/estatistica/populacao/condicaodevida/pof/2003medidas/pof2003medidas.pdf

19. Leão LS, Araújo LM, Moraes LT, Assis AM. Prevalência de obesidade em escolares de Salvador, Bahia. Arq Bras Endocrinol Metab 2003;47:151-7.

20. Escrivão MA, Oliveira FL, Taddei JA, Lopez FA. Obesidade exógena na infância e na adolescência. J Pediatr (Rio J) 2000;76:S305-10.

21. Monteiro CA, Mondini L, Souza ALM, Popkin BN. Da desnutrição para a obesidade: a transição nutricional no Brasil. In: Monteiro CA, editor. Velhos e novos males da saúde no Brasil: a evolução do país e suas doenças. $1^{\text {a }}$ ed. São Paulo: Hucitec; 1995. p. 247-55.

22. Vieweg VR, Johnston CH, Lanier JO, FernandezA, Pandurangi AK. Correlation between high risk obesity groups and low socioeconomic status in school children. South Med J 2007;100:8-13. 